\title{
POINTING BIAS CALIBRATION OF GAOFEN-7 LASER ALTIMETER BASED ON SINGLE LASER FOOTPRINT IMAGE
}

\author{
Junfeng Xie ${ }^{1,3}$, Ren $\mathrm{Liu}^{2 *}$, Fan $\mathrm{Mo}^{1}$, Hongzhao Tang ${ }^{1}$,Huihui Jiao ${ }^{3}$, Yongkang $\mathrm{Mei}^{1}$ and Chenchen Yang ${ }^{1}$ \\ ${ }^{1}$ Land Satellite Remote Sensing Application Center, Ministry of Natural Resources of P.R.China, Beijing, China - (junfeng_xie, \\ surveymofan)@163.com, tanghz@lasac.cn,(362151647, 571200645)@qq.com \\ ${ }^{2}$ School of earth sciences and engineering, Hohai university, Nanjing, China - rs_liur@163.com \\ ${ }^{3}$ School of Geomatics, Liaoning Technical University, Fuxin, China - $920770527 @$ qq.com
}

\section{Commission II, WG II/1}

KEY WORDS: GaoFen-7 satellite, laser altimeter, laser footprint image, in-orbit calibration, geolocation model, AW3D30 DSM

\begin{abstract}
:
The GaoFen-7 (GF-7) satellite is successfully launched on November 3, 2019, and its laser altimeter system is officially and firstly employed as the main payload for earth observations in China, which includes two sets of laser altimeters and laser footprint cameras. The Laser Footprint Image (LFI) is used to capture laser spots on the ground. In order to make up for the shortcomings of high cost field work for the traditional laser altimeter ground detector-based calibration method, this paper proposes a novel laser altimeter calibration method based on LFI. Firstly, the spaceborne laser calibration model and the Laser Footprint Camera (LFC) geolocation model are established. Secondly, the image coordinates of laser spot centroid are extracted from LFI, and the ground location of is obtained by ray intersecting with the reference Digital Surface Model (DSM). Finally, the centroid of laser spot is considered as Ground Control Point (GCP), and the pointing bias of GF-7 laser altimeter is calibrated by the Least Squares Estimation (LSE). The ALOS Global Digital Surface Model "ALOS World 3D-30m" (AW3D30) is used to evaluate the elevation accuracy of GF-7 laser altimeter before and after the calibration. The results indicate that elevation accuracy of the GF-7 laser altimeter is improved significantly after calibration. The proposed method can be effectively applied for high-frequency geometric calibration of GF-7 laser altimeter.
\end{abstract}

\section{INTRODUCTION}

GF-7 Satellite was successfully launched on November 3, 2019, which is equipped with the Chinese first official spaceborne laser altimeter system for Earth observation. The satellite operates in a solar synchronous orbit at an altitude of $505 \mathrm{~km}$. The two linear array optical cameras and two laser altimeters mounted on the satellite can respectively acquire high spatial resolution stereo mapping remote sensing data and high precision laser data. They are mainly applied for 1:10000 stereo mapping and larger scale geographic information update.

The GF-7 laser altimeter system employs dual beam laser to measure the range from emitting point to the earth surface, and each beam includes two laser altimeters, the primary and the backup payload. Meanwhile, it equips with two LFCs and a Laser Optical Axis Surveillance Camera (LOASC). The LFC can take a panchromatic image, and capture the shape of laser spot and the features of ground object (Tang et al., 2019b). The laser altimetry system is mainly used to obtain accurate GCPs and assist the optical stereo image to improve the elevation accuracy of products. However, the pointing, ranging and other parameters of the laser altimeter will change due to platform vibration during launching, and environmental changes after launching, which may reduce the measure accuracy of laser spot. So, it is necessary to in-orbit calibrate these systematic errors when satellite operates.

According to the different types of reference data, the in-orbit geometric calibration method for spaceborne laser altimeter mainly includes the ground-based infrared detector calibration, airborne infrared camera imaging calibration, Corner Cube Retroreflectors (CCR) calibration, attitude-maneuver calibration and surface matching calibration. The ground-based infrared detector calibration method has high accuracy, it needs to design and deploy a large number of detector arrays before the satellite flew across the test site, which consumes a lot of manpower and is not suitable for high-frequency calibration (Magruder et al., 2003a; Magruder et al., 2003b; Xie et al., 2018; Yi Hong, 2017). Airborne infrared camera imaging calibration require strict synchronous exposure of spaceborne laser and airborne camera, and can only be implemented at night when the moon is below the horizon (Magruder et al., 2010; Schutz, 2001). The CCR calibration method mainly relies on the waveform to extract the echo energy, and the atmosphere and reflectivity of ground objects will introduce errors (Ma et al., 2018; Magruder et al., 2006). The attitude-maneuver calibration method requires high-agility satellite platform, and will introduce additional high-frequency attitude noise (Luthcke et al., 2000; Luthcke et al., 2005). Terrain matching calibration method relies on high-precision reference DSM, so it is necessary to select special terrain for test (F. Martin et al., 2005; Tang et al., 2019a).

In this paper, we will make full use of the LFC carried by the GF-7, and propose a novel geometric calibration method of laser altimeter based on single LFC image. Firstly, the image coordinates of the laser spot in the LFI is extracted, and then the spaceborne laser geometric calibration model and LFC geolocation model are established. The laser spot is geolocated by the ray resection with single LFI and the reference DSM data. This location of the laser spot centroid on the ground is employed as GCPs. Finally, the pointing bias of GF-7 laser can be calibrated. The ellipsoidal height of more than 2100 laser points in the $153^{\text {th }}, 246^{\text {th }}$ and $755^{\text {th }}$ track of GF-7 satellite is calculated and evaluated before and after calibration. The validity of the proposed method is verified by the elevation accuracy comparison between laser geolocation result and 
ALOS Global DSM-AW3D30 (Takaku et al., 2016a; Takaku et al., 2016b).

\section{GF-7 LASER ALTIMETER SYSTEM}

The spaceborne laser altimeter system of GF-7 mainly consists of two sets of laser altimeters (dual laser per set, the primary and the backup), which include a dual-beam simultaneous laser for earth observation, and an LOASC and a receiving telescope with an aperture of $0.6 \mathrm{~m}$. The working principle diagram of GF7 laser altimeter is shown figure 1 .

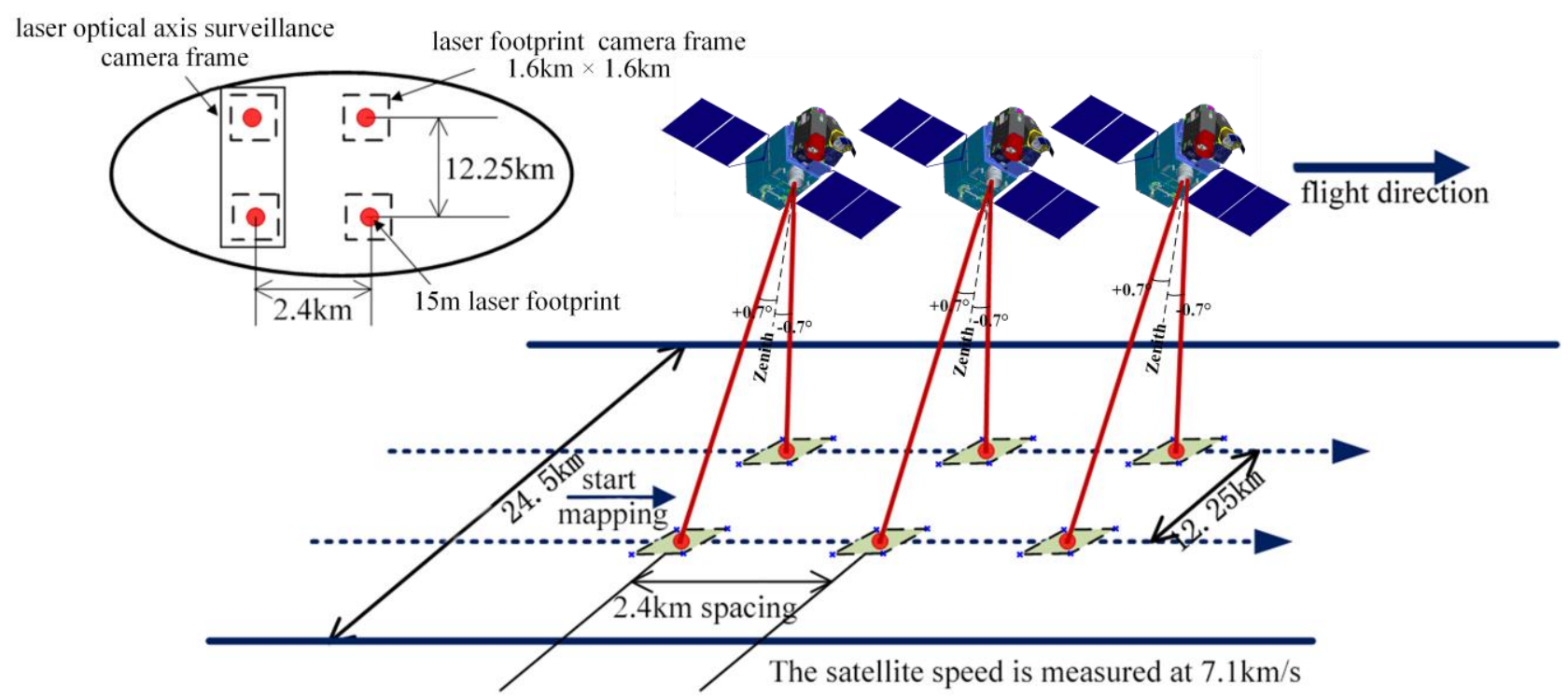

Figure 1. Schematic diagram of the GF-7 spaceborne laser altimeter system

The spaceborne laser altimeter system of GF-7 consists of two sets of lasers, which emit laser pulses to the ground at the frequency of $3 \mathrm{HZ}$ to generate two beams laser defined as beam 1 and beam 2 . And the zenith direction angle between each beam and the satellite nadir pointing is 0.7 degrees. When two laser beams of GF-7 penetrate the atmosphere and reach the ground, two laser spots with a diameter of about $15 \mathrm{~m}$ are formed, and the echo waveforms of the ground objects in the spot are recorded by sampling points. The basic parameters of GF-7 spaceborne laser altimeter are shown in table 1 .

\begin{tabular}{|l|c|}
\hline Parameter & Value \\
\hline Number of beams & 2 \\
Laser Wavelength & $1064 \mathrm{~nm}$ \\
Laser energy & $100 \sim 180 \mathrm{~mJ}$ (adjustable) \\
Emission pulse width & $4 \sim 8 \mathrm{~ns}$ \\
Laser divergence angle & $30 \sim 40 \mu \mathrm{rad}$ \\
Receiving telescope aperture & $600 \mathrm{~mm}$ \\
Pulse repetition frequency & $3 / 6 \mathrm{~Hz}$ (adjustable) \\
Echo digitization interval & $0.5 \mathrm{~ns}$ \\
Laser emission efficiency & 0.994 \\
Laser receiving efficiency & 0.790 \\
Laser ranging range & $450 \sim 550 \mathrm{~km}$ \\
Laser ranging accuracy & $\leq 0.3 \mathrm{~m}\left(\right.$ Slope $\left.<15^{\circ}\right)$ \\
\hline
\end{tabular}

Table 1. Basic parameters of the GF-7 laser altimeter

The LFC of GF-7 is a frame camera. The image size of LFI is $550 \times 550$ pixel. The image resolution is about $3.2 \mathrm{~m}$. This image can record the laser spot and the ground objects information around the footprint at the time of laser emission. The two LFCs correspond to two laser beams. LFC1 serves for Beam 1, and LFC2 is responsible for Beam 2. There are two types of imaging modes: synchronous mode and asynchronous mode (Tang et al., 2019b). The experiments adopt the synchronous mode to obtain the LFIs. The parameters of the LFCs are shown in table 2.

\begin{tabular}{|l|c|}
\hline Parameters & LFC \\
\hline spectral range & Visible light: $500-700 \mathrm{~nm}$ \\
Instantaneous Field of View & laser: $1064 \mathrm{~nm}$ \\
Modulation Transfer Function & $6.4 \mu \mathrm{rad}$ \\
(MTF) & $\geq 0.20$ \\
Pixel size & $16.5 \mu \mathrm{m}$ \\
Image size & $550 \times 550$ pixels \\
Field of View & $\pm 0.1^{\circ}$ \\
Optical aperture & $600 \mathrm{~mm}$ \\
Digitalizing bit & $\geq 10 \mathrm{bit}$ \\
Optical efficiency & $\geq 70 \%$ \\
Principal distance & LFC $1: 2580.2 \mathrm{~mm}$ \\
\end{tabular}

Table 2. Basic parameters of the GF-7 LFC

\section{SPACEBORNE LASER ALTIMETER CALIBRATION}

The laser spot and ground feature can be displayed in the same image with the synchronous exposure mode of LFC. The image coordinates of laser spot centroid can be obtained from LFC image, which be converted to ground object coordinates by LFC geolocation model. It is applied as GCP in GF-7 laser geometric calibration model to get the optimal pointing.

\subsection{Laser Spot Geolocation based on LFC}

\section{1) Laser Spot Image Centroiding of LFI}

Firstly, laser spot is a blur ellipse composed of multiple pixels on LFI, and its energy is reflected by brightness. The energy of GF-7 laser spot has a gaussian distribution on LFI, as shown in figure 2. The laser spot size is magnified four times by the beam expander. The maximum scope of GF-7 laser spot is about 20 pixels on figure2 LFI. The actual diameter of GF-7 laser spot is approximately equal to $15 \mathrm{~m}$ (four pixels). Meanwhile, in figure 4 , there is another laser spot on the image edge, which is reflected by another side surface of the prism. 


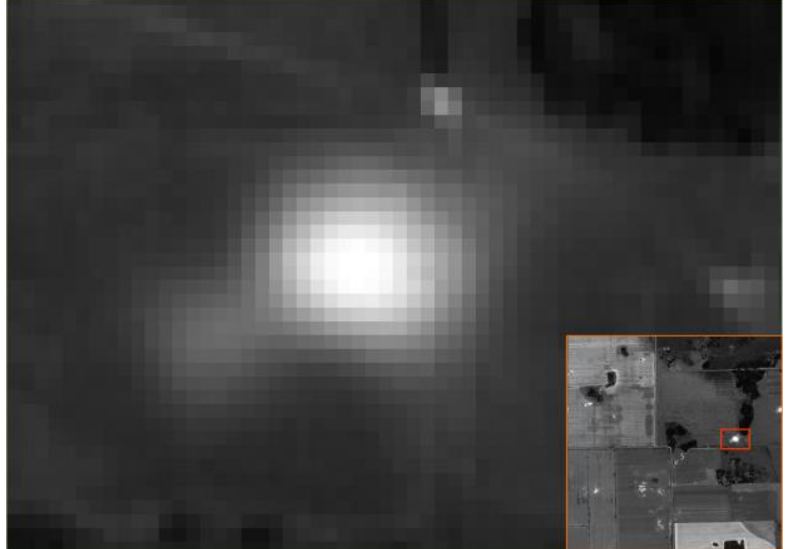

Figure 2. The laser spot of GF-7 beam 2 on LFI

The grayscale weighting method can be used to extract the centroid of GF-7 laser spot, which accurately determine the image coordinate of the laser centroid. The grayscale weighting formula is shown below.

$$
g(x, y)=\frac{\sum_{s=-a}^{a} \sum_{t=-b}^{b} w(s, t) f(x+s, y+t)}{\sum_{s=-a}^{a} \sum_{t=-b}^{b} w(s, t)}
$$

Where $g(x, y)$ is the LFI coordinate of laser spot centroid; $w(s, t)$ is the image grayscale value at position $(s, t)$; $f(x+s, y+t)$ represents the row and column coordinates at position $(s, t) ; \mathrm{a}, \mathrm{b}$ are half of the laser spot rectangle row and column.

Secondly, since the optical axis of LFC and laser are not parallel, so the original position of GF-7 laser spot in the LFC image is not the actual position. The converting formula involving the original and actual position of the laser spot can be obtained by the laboratory test, and its measurement error is better than 0.2 pixels. The formula is given below.

$$
P(x, y)=P_{0}\left(x_{0}, y_{0}\right)+\Delta d \cos \alpha
$$

Where $P(x, y)$ is the actual position of laser in LFC image; $P\left(x_{0}, y_{0}\right)$ is the original position of laser in LFC image; $\Delta d$ is the distance between $P(x, y)$ and $P\left(x_{0}, y_{0}\right)$ measured in laboratory; $\alpha$ is the azimuth angle between $P(x, y)$ and $P\left(x_{0}, y_{0}\right)$ measured in laboratory.

\section{2) Geolocation of Laser Spot}

Based on the imaging theory of the area-array camera, the LFC geolocation model can be established, which is shown as below.

$$
\left[\begin{array}{c}
X_{g} \\
Y_{g} \\
Z_{g}
\end{array}\right]=\left[\begin{array}{c}
X_{s} \\
Y_{s} \\
Z_{s}
\end{array}\right]+\lambda R_{I C R F}^{I T R F} R_{B O D}^{I C R F}(\text { pitch }, \text { roll, yaw }) \cdot R_{C A M}^{B O D}\left[\begin{array}{c}
\tan \left[\psi_{x}(s, 1)\right] \\
\tan \left[\psi_{y}(s, 1)\right] \\
-1
\end{array}\right]
$$

Where $(s, l)$ is the detector location in the image coordinate system; $\psi_{x}$ and $\psi_{y}$ are pointing angles of each detector in CMOS (Complementary Metal Oxide Semiconductor) camera; $R_{C A M}^{B O D}$ is the rotation matrix from the LFC to satellite body; (pitch, roll,yaw) is the attitude of the LFC; $R_{B O D}^{I C R F}$ is the attitude rotation matrix; $R_{I C R F}^{I T R F}$ represents the rotation matrix from the ICRF to the International Terrestrial Reference Frame
(ITRF); $\lambda$ is the scale factor; $\left[\begin{array}{lll}\mathrm{X}_{s} & Y_{s} & Z_{s}\end{array}\right]^{T}$ represents the coordinates of the projection centre in the ITRF.

\section{3) Laser Geolocation based on DSM and Single LFI}

When the image coordinates of the laser spot centroid in the LFC image are obtained, the actual image coordinates can be acquired by the formula (2). With the image coordinates of the actual laser spot and the internal and external elements of the LFC, the ray equation of the laser spot is founded by formula (3). The ray is intersected with the DSM data, and the geographic location of the laser spot is obtained by iterative estimation. The specific steps are shown in the figure 3 .

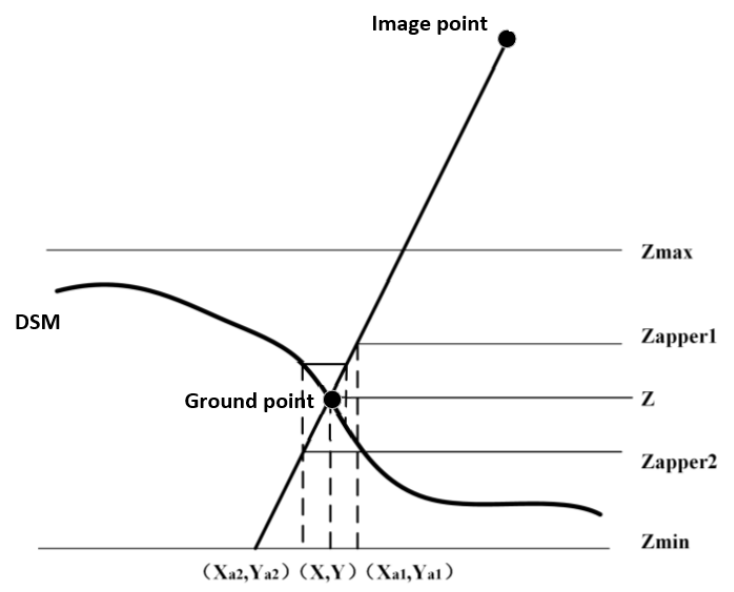

Figure 3. Schematic diagram of laser geometric positioning of single footprint image assisted by DSM

1) Setting the maximum elevation and minimum elevation range according to reference DSM. Assuming the average elevation be zappr1, we can get horizontal coordinates $\left(\mathrm{X}_{\mathrm{a} 1}, \mathrm{Y}_{\mathrm{a} 1}\right)$ by the intersection of ray equation with an elevation plane zappr1.

2) Interpolating the horizontal coordinates $\left(\mathrm{X}_{\mathrm{a} 1}, \mathrm{Y}_{\mathrm{a} 1}\right)$ into DSM, then new elevation plane Zappr2 is generated.

3) Repeatedly, new horizontal coordinates $\left(\mathrm{X}_{\mathrm{a} 2}, \mathrm{Y}_{\mathrm{a} 2}\right)$ can got by intersecting zappr2 and ray equation. Then, the next elevation plane is obtained by interpolation with DSM.

4) The geographic location $(X, Y, Z)$ of the laser point can be obtained by iterative estimation.

\subsection{Geometric Calibration Model of Laser Altimeter}

According to the geometric relationship between laser, satellite platform and earth ellipsoid, the spaceborne laser geometric calibration model is established by using satellite attitude, orbit and the geometric relationship between laser and satellite platform, its matrix form is shown below.

$$
\begin{aligned}
&\left(\begin{array}{c}
\mathrm{X}_{\text {spot }} \\
\mathrm{Y}_{\text {spot }}= \\
\mathrm{Z}_{\text {spot }}
\end{array}\right)_{\text {ITRF }}\left(\begin{array}{c}
\mathrm{X}_{\mathrm{s}} \\
\mathrm{Y}_{\mathrm{s}} \\
\mathrm{Z}_{\mathrm{s}}
\end{array}\right)_{\text {ITRF }}+\mathrm{R}_{\mathrm{ICRF}}^{\mathrm{ITRF}} \mathrm{R}_{\mathrm{BOD}}^{\mathrm{ICRF}} \\
& \cdot\left(\left(\begin{array}{c}
\Delta \mathrm{X}_{\mathrm{ref}} \\
\Delta \mathrm{Y}_{\text {ref }} \\
\Delta \mathrm{Z}_{\mathrm{ref}}
\end{array}\right)_{\mathrm{BOD}}+\rho_{0}(\mathrm{t})\left(\begin{array}{c}
\cos \beta \cos \alpha \\
\cos \beta \sin \alpha \\
\sin \beta
\end{array}\right)_{\mathrm{BOD}}\right]
\end{aligned}
$$

where $\left(X_{s p o t}, Y_{s p o t}, Z_{s p o t}\right)_{I T R F}^{T}$ is the location of the laser footprint in ITRF; $\left(X_{s}, Y_{s}, Z_{s}\right)_{I T R F}^{T}$ is the position of the satellite centroid in the ITRF; $R_{B O D}^{I C R F}$ is the rotation matrix of the satellite body coordinate system in reference to ICRF; $R_{I C R F}^{I T R F}$ is the rotation 
matrix from the ICRF to the ITRF; $\left(\Delta X_{r e f}, \Delta Y_{r e f}, \Delta Z_{r e f}\right)_{B O D}^{T}$ is the offset between the laser emission reference point and the centre of the satellite mass in the satellite body coordinate system; $\rho_{0}(t)$ is the laser range; $\alpha$ and $\beta$ are the pointing angles from the laser altimeter to satellite body frame.

According to formula (4), the distance error between laser emission reference point and laser spot can be expressed as:

$\Delta \rho=\sqrt{\left(\frac{a 1-\Delta X_{r e f}}{\cos \beta \cos \alpha}\right)^{2}+\left(\frac{b_{1}-\Delta Y_{r e f}}{\cos \beta \sin \alpha}\right)^{2}+\left(\frac{c_{1}-\Delta Z_{r e f}}{\sin \beta}\right)^{2}}-\rho_{0}$

Where, $\left(\begin{array}{l}a_{1} \\ b_{1} \\ c_{1}\end{array}\right)=\left(R_{I C R F}^{I T R F} R_{B O D}^{I C R F}\right)^{-1}\left(\begin{array}{c}X_{s p o t}-X_{s} \\ Y_{s p o t}-Y_{s} \\ Z_{s p o t}-Z_{s}\end{array}\right) \quad, \quad \rho_{0}$ is the ranging

value calculated with laser pulse transit time.

According to formula 4, the error equation is derived as:

$$
V=A X-L
$$

Where $A=\left(\begin{array}{ll}\frac{\partial \rho}{\partial \alpha} & \frac{\partial \rho}{\partial \beta}\end{array}\right), L=\rho_{0}, X=\left(\begin{array}{ll}d \alpha & d \beta\end{array}\right)$.

Lastly, according to the criterion of the least ranging error from the emission point of the laser to the ground spot, the GF-7 laser pointing bias are solved by least squares estimation, and the weighting matrix here is an identity matrix.

\section{EXPERIMENT}

\subsection{Test Data}

For the GF-7 satellite, the laser altimeter operates about 8 tracks a day, and each track lasts at most 15 minutes. So far, hundreds of tracks of laser data had been downlinked to the ground. The terrain of the test area should be flat and there is no cloud over the area to ensure that laser footprint can clearly display the information of the ground objects and laser spots. Therefore, the laser data of the $755^{\text {th }}$ track passing over South America on December 23, 2019 was selected as test data. And the effective laser data of the $755^{\text {th }}$ track is shown in the figure $3 \mathrm{a}$. The cloudless LFI in $755^{\text {th }}$ track of GF-7 satellite is selected as calibration data, which located on farmland in Reconquista Argentina (Figure 3b). The calibration data includes two set laser data (laser data of beam 1 and beam 2) and a pair of LFC images (LFIs of beams 1 and 2), and both of their time codes are $188518622.33 \mathrm{~s}$. The time code is the accumulated seconds since January 1, 2014.
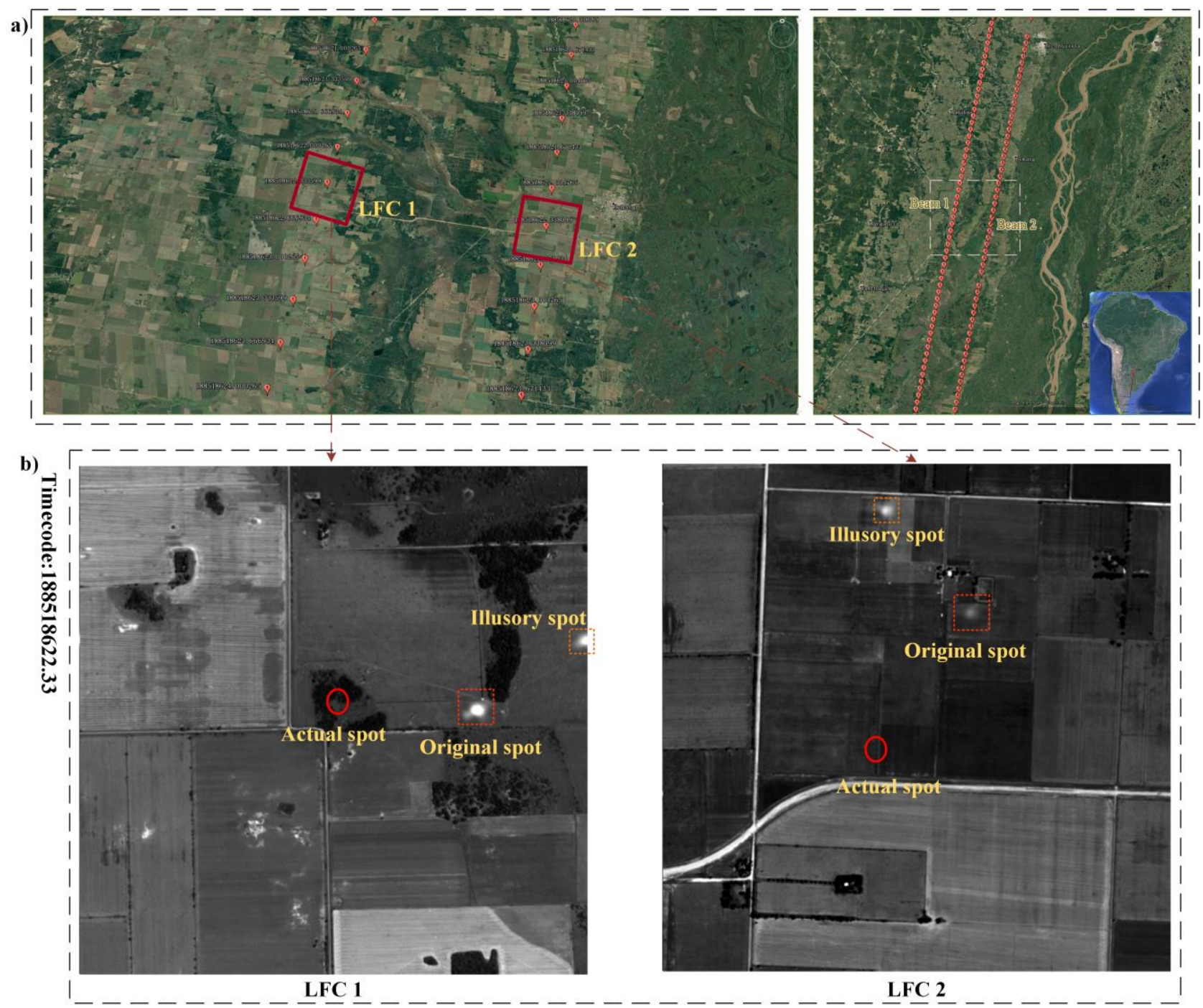

Figure 4. Laser data and LFI in the $755^{\text {th }}$ track (Imaging timecode: $188518622.33 \mathrm{~s}$ ) 
The $755^{\text {th }}, 153^{\text {th }}$ and $246^{\text {th }}$ track laser data are used to verify the laser elevation accuracy before and after calibration. The related information about these three tracks of laser data is shown in table 3. The number of effective pulses is the total number of laser pulses minus the number of points of the laser without echo. Several laser points whose echo backward from the cloud layer are defined as invalid data, and the laser points after excluding invalid points is used for test.

\begin{tabular}{|c|c|c|c|c|}
\hline $\begin{array}{c}\text { Track } \\
\text { number }\end{array}$ & Beam & $\begin{array}{c}\text { Total } \\
\text { pulses }\end{array}$ & $\begin{array}{c}\text { Effective } \\
\text { pulses }\end{array}$ & $\begin{array}{c}\text { Test laser } \\
\text { points }\end{array}$ \\
\hline \multirow{2}{*}{153} & Beam 1 & 436 & 314 & 210 \\
& Beam 2 & 436 & 256 & 187 \\
246 & Beam 1 & 342 & 293 & 293 \\
& Beam 2 & 342 & 292 & 292 \\
755 & Beam 1 & 928 & 703 & 604 \\
& Beam 2 & 928 & 685 & 579 \\
\hline
\end{tabular}

Table 3. The statistical information of three track laser data

AW3D30 DSM, whose elevation accuracy is 5 meters (Takaku et al., 2016a), is used as reference terrain data for laser elevation accuracy verification before and after calibration. The image of AW3D30 DSM is shown in Figure 3, and its latitude and longitude range are $29^{\circ}-30^{\circ} \mathrm{S}$ and $59^{\circ}-60^{\circ} \mathrm{W}$.

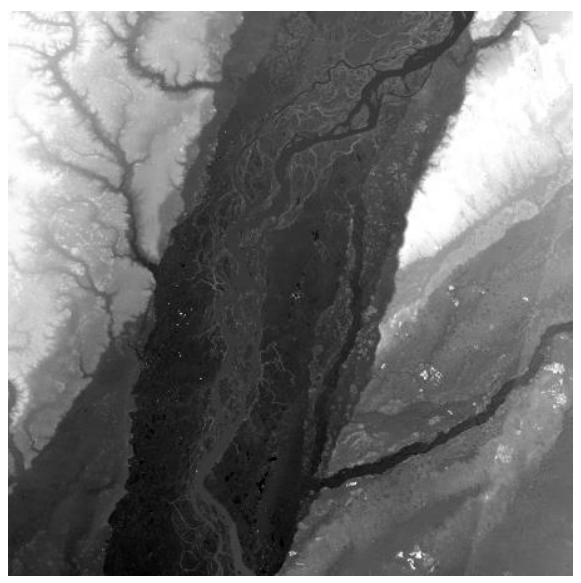

Figure 5. AW3D30 product covering Laser data of the $755^{\text {th }}$ track

\subsection{Result and analysis}

Considering the misalignment angle between GF-7 beam 1 and beam 2 is unstable, the pointing bias calibration of the two beams are treated independently. Firstly, for beam 1, the image coordinate of initial centroid $P_{0}$ (x:430.557,y:264.489)(unit: pixel) on the LFC image as shown in Figure 3 (b) are extracted, and actual centroid image coordinates $P(\mathrm{x}: 267.649, \mathrm{y}: 254.344)$ (unit: pixel) are obtained by formula (2).

Then, we substituted $P$ into formula (3) to get initial geographic planimetric coordinate (B: -29.488714, L: -59.920395) (unit: degree) of laser footprint centroid while the AW3D30 data covering this laser footprint was selected as reference DSM. The ray equation in formula (3) is intersected with the reference DSM by iterative estimation to obtain the optimal geographic coordinate (B: $-29.488883^{\circ}$, L: $-59.920461^{\circ}, \mathrm{H}: 54.15 \mathrm{~m}$ ) of laser spot, which is expressed in WGS84.

Lastly, the laser optimal location is taken into formula (4), based on the satellite attitude and orbit data, the GF-7 beam 1 pointing bias are calibrated based on the tidal and atmospheric correction. Beam 2 is the same as beam 1 . The pointing bias $(\Delta \alpha, \Delta \beta)$ between initial and calibrated pointing angle are shown in table 4 .

\begin{tabular}{|c|c|c|}
\hline \multirow{2}{*}{ Beam } & \multicolumn{2}{|c|}{ Pointing bias } \\
\cline { 2 - 3 } & $\Delta \alpha /^{\circ}$ & $\Delta \beta /^{\circ}$ \\
\hline Beam 1 & 0.01244 & -0.01549 \\
Beam 2 & 0.08892 & -0.02248 \\
\hline
\end{tabular}

Table 4 . The pointing bias of GF-7 beam 1 and beam 2

The $153^{\text {th }}, 246^{\text {th }}$ and $755^{\text {th }}$ track laser data are employed to evaluate the laser elevation accuracy before and after calibration. The elevation difference values (Delta_H) between the laser and AW3D30 DSM are calculated before and after the calibration. The results are shown in figure6 11 below.

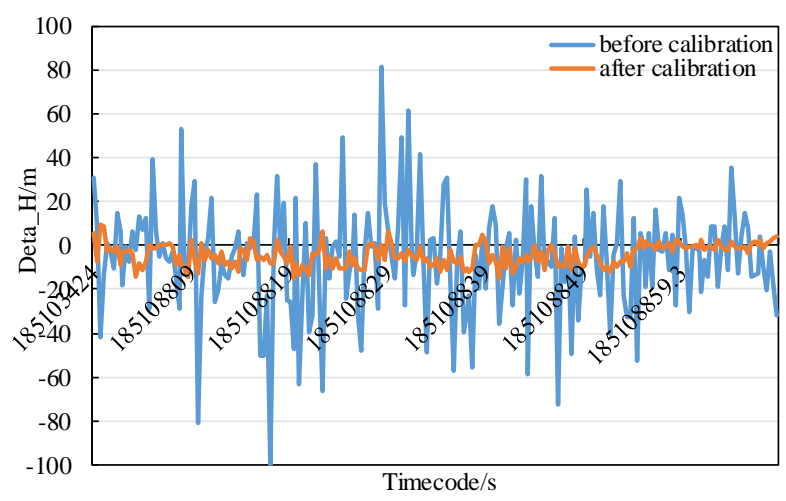

Figure 6. Elevation difference of the $153^{\text {th }}$ track laser data (Beam 1)

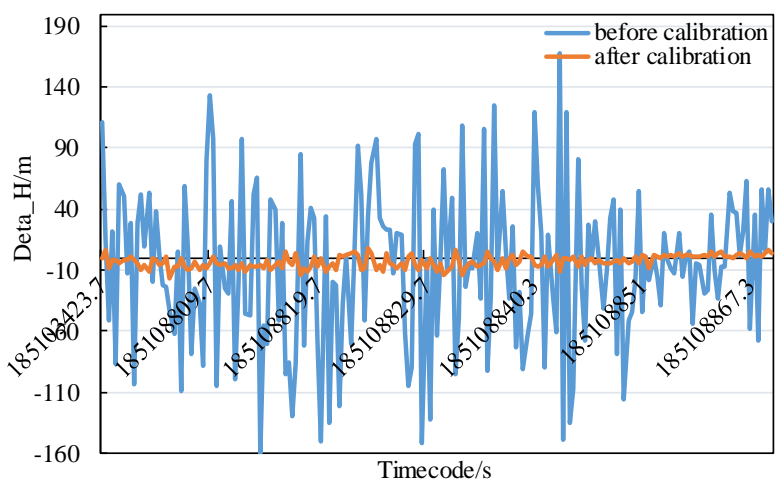

Figure 7. Elevation difference of the $153^{\text {th }}$ track laser data (Beam 2)

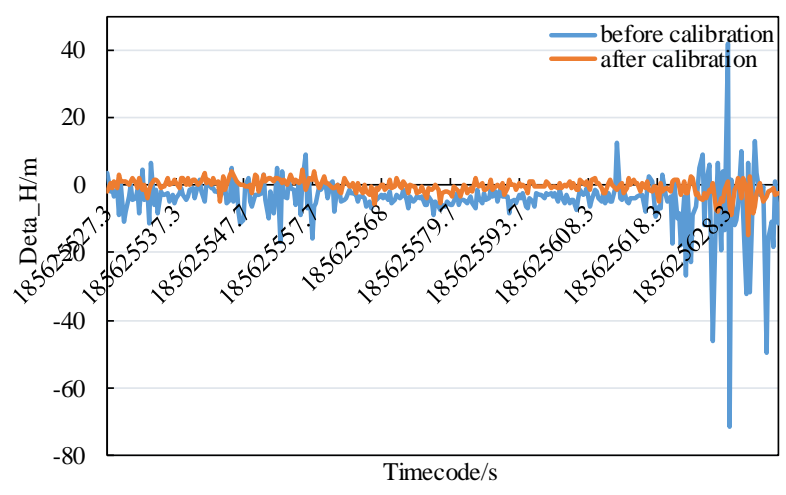

Figure 8 . Elevation difference of the $246^{\text {th }}$ track laser data (Beam 1) 


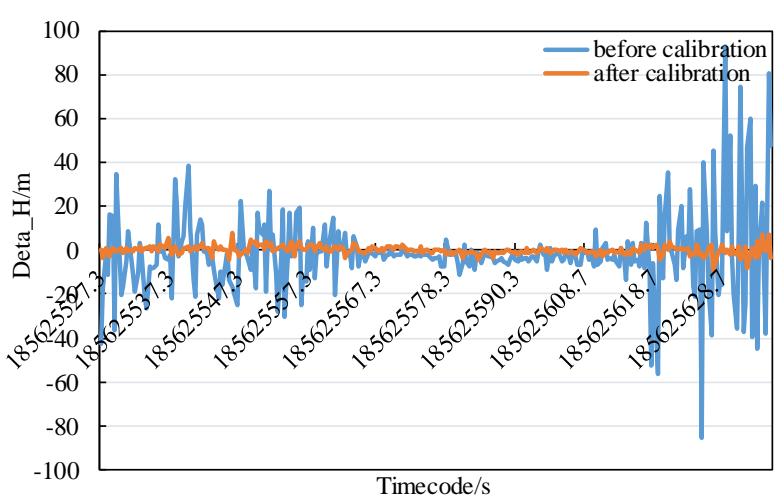

Figure 9. Elevation difference of the $246^{\text {th }}$ track laser data (Beam 2)

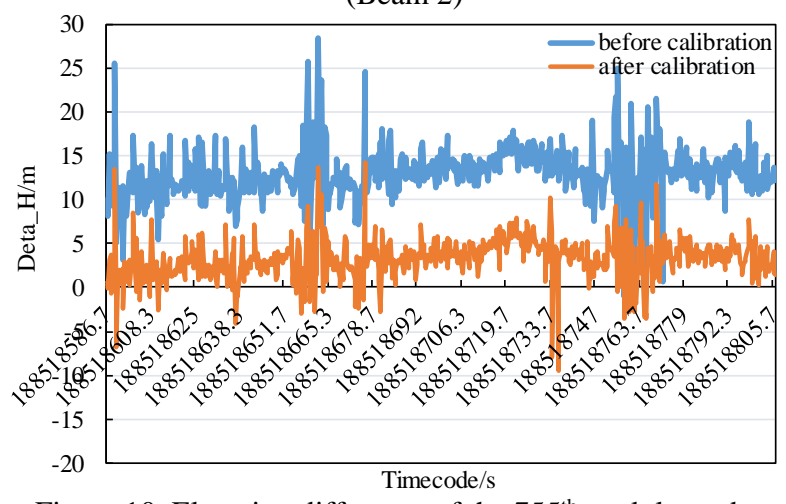

Figure 10. Elevation difference of the $755^{\text {th }}$ track laser data (Beam 1)

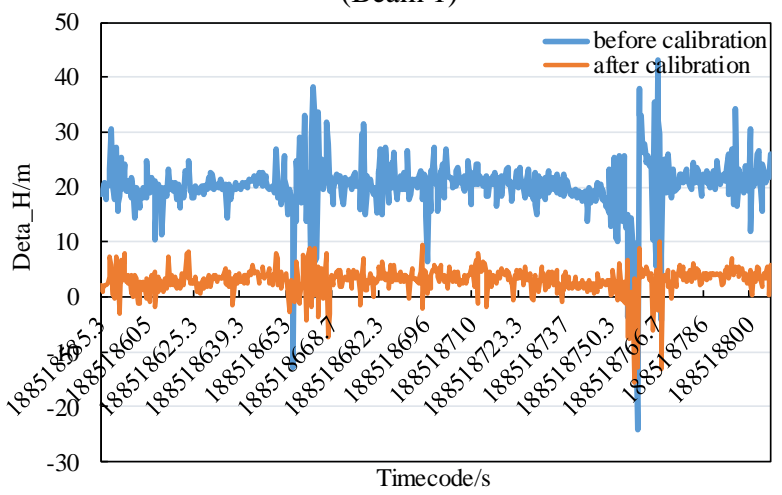

Figure 11. Elevation difference of the $755^{\text {th }}$ track laser data (Beam 2)

The result indicates that the elevation accuracy of GF-7 laser altimeter after calibration is significantly improved compared with that before calibration. The laser elevation error of some tracks is more than $100 \mathrm{~m}$ without calibration. There are constant values at the beginning of figure 8,9 and 11 . The reason for that is the terrain of these track beginning is flat, and the terrain gradually turns into mountains in the satellites flight direction. The same horizontal position deviation of laser footprint in flat area will cause the less elevation error compared with that in mountainous area.

In order to quantify elevation accuracy after calibration, the mean and Root Mean Square Error (RMSE) of elevation difference before and after calibration are shown in table 5,6.

\begin{tabular}{|c|c|c|c|c|}
\hline \multirow{3}{*}{ Track number } & \multicolumn{2}{|c|}{ Before calibration } & \multicolumn{2}{c|}{ After calibration } \\
\cline { 2 - 5 } & $\begin{array}{c}\text { Mean } \\
\text { /m }\end{array}$ & $\begin{array}{c}\text { RMSE } \\
/ \mathrm{m}\end{array}$ & $\begin{array}{c}\text { Mean } \\
/ \mathrm{m}\end{array}$ & $\begin{array}{c}\text { RMSE } \\
/ \mathrm{m}\end{array}$ \\
\hline 153 & 6.16 & 24.55 & 4.35 & 4.95 \\
246 & 4.34 & 8.01 & 0.46 & 2.22 \\
\hline
\end{tabular}

\begin{tabular}{|c|c|c|c|c|}
\hline 755 & 12.86 & 3.06 & 3.23 & 2.41 \\
Mean & 7.79 & 11.87 & 2.68 & 3.19 \\
\hline
\end{tabular}

Table 5. The mean and RMSE of GF-7 Beam 1 elevation difference

\begin{tabular}{|c|c|c|c|c|}
\hline \multirow{3}{*}{ Track number } & \multicolumn{2}{|c|}{ Before calibration } & \multicolumn{2}{c|}{ After calibration } \\
\cline { 2 - 5 } & $\begin{array}{c}\text { Mean } \\
/ \mathrm{m}\end{array}$ & $\begin{array}{c}\text { RMSE } \\
/ \mathrm{m}\end{array}$ & $\begin{array}{c}\text { Mean } \\
/ \mathrm{m}\end{array}$ & $\begin{array}{c}\text { RMSE } \\
/ \mathrm{m}\end{array}$ \\
\hline 153 & 7.28 & 63.66 & 3.02 & 5.06 \\
246 & 1.86 & 17.93 & 0.03 & 2.04 \\
755 & 20.29 & 5.12 & 3.03 & 2.45 \\
Mean & 9.81 & 28.90 & 2.03 & 3.18 \\
\hline
\end{tabular}

Table 6. The Mean and RMSE of elevation difference (Beam 2)

According to the above results, before calibration, GF-7 laser beam 1 elevation accuracy is significantly better than Beam 2 . After calibration, the elevation accuracy of the GF-7 laser altimeter has been improved by more than four times. The elevation accuracy of beam 1 was improved from $7.79 \mathrm{~m} \pm$ $11.87 \mathrm{~m}$ to $2.68 \mathrm{~m} \pm 3.19 \mathrm{~m}$, and the laser elevation accuracy of beam 2 was improved from $9.81 \mathrm{~m} \pm 28.90 \mathrm{~m}$ to $2.03 \mathrm{~m} \pm 3.18$ $\mathrm{m}$. From figure 6-11, we can see that the elevation accuracy is relatively stable after calibration, and its variation tend fluctuate gently. And the elevation difference of the laser points is relatively large before calibration in above figures. The main reason is the pointing bias can still introduce the larger elevation errors in mountainous terrain.

\section{CONCLUSION}

In this paper, we propose an in-orbit pointing bias calibration method for GF-7 laser altimeter based on single footprint image. More than 2100 effective laser points of the $153^{\text {th }}, 246^{\text {th }}$ and $755^{\text {th }}$ track were employed to validate the calibration accuracy. Taking AW3D30 data as reference DSM, after calibration, the elevation accuracy of GF-7 laser beam 1 and beam 2 were improved by at least four times compared with that before calibration. And the GF-7 laser elevation difference changes are quite stable after calibration. The results show that the proposed calibration method is effective and feasible.

Although the elevation accuracy of the GF7 laser has improved significantly after calibration, the GF-7 laser maximum elevation error after calibration is still close to $5 \mathrm{~m}$ from table 5 and 6. On the one hand, it is related with the elevation accuracy of reference DSM. The AW3D30 had been used as reference DSM, its elevation accuracy is within 5 meters. So, the calibration accuracy is limited by reference DSM. On the other hand, AW3D30 is used for accuracy evaluation, and the elevation accuracy after calibration is close to ALOS 30 DSM accuracy.

In the future, the LFC parameters will be calibrated, and based on which the test result with proposed method will be better with higher-accuracy terrain data. And, the location of the laser spot on the ground will be measured by RTK to evaluate the absolute elevation accuracy of GF-7 laser altimeter. Meanwhile, as the calibration times increases, the stability of laser pointing with time will be analysed.

\section{ACKNOWLEDGEMENTS}

This study was financially supported in part by the High Resolution Remote Sensing, Surveying and Mapping Application Program under Grant 42-Y30B04-9001-19/21, in part by the Authenticity Validation Technology of Elevation Accuracy of the GF-7 Laser Altimeter under Grant 42-Y20A119001-17/18, Active and Passive Composite Mapping and Application Technology with Visible, Infrared and Laser 
Sensors under Grant D040106, and Multi-beam Terrain Detection Laser and its Application Technology (D040105), in part by the National Natural Science Foundation of China under Project Nos. 41571440, 41771360, 41971426, and in part by Class B Project of Beijing Science and Technology Association Jinqiao Project Seed Fund (No. ZZ19013).

\section{REFERENCES}

F. Martin, C., Thomas, R.H., Krabill, W.B., Manizade, S.S., 2005. ICESat range and mounting bias estimation over precisely-surveyed terrain. Geophysical Research Letters 32, L21S07.

Luthcke, S.B., Rowlands, D.D., Mccarthy, J.J., Pavlis, D.E., Stoneking, E., 2000. Spaceborne Laser-Altimeter-Pointing Bias Calibration from Range Residual Analysis. Journal of Spacecraft \& Rockets 37(3), 374-384.

Luthcke, S.B., Rowlands, D.D., Williams, T.A., Sirota, M., 2005. Reduction of ICESat systematic geolocation errors and the impact on ice sheet elevation change detection. Geophysical Research Letters 32(21), 312-321.

Ma, Y., Li, S., Wu, Y., Liu, R., Wang, X.H., Ma, X., 2018. Method for determining the footprint center of a satellite laser altimeter based on marked waveforms by CCRs. Applied Optics 57(30), 8928.

Magruder, L.A., Ricklefs, R.L., Silverberg, E.C., Horstman, M.F., Suleman, M.A., Schutz, B.E., 2010. ICESat Geolocation Validation Using Airborne Photography. IEEE Transactions on Geoscience \& Remote Sensing 48(6), 2758-2766.

Magruder, L.A., Schutz, B.E., Silverberg, E.C., 2003a. Laser pointing angle and time of measurement verification of the ICESat laser altimeter using a ground-based electro-optical detection system. Journal of Geodesy 77(3), 148-154.

Magruder, L.A., Suleman, M.A., Schutz, B.E., 2003b. ICESat laser altimeter measurement time validation system. Measurement Science and Technology 14(11), 1978-1985.

Magruder, L.A., Webb, C.E., Urban, T.J., Silverberg, E.C., Schutz, B.E., 2006. ICESat Altimetry Data Product Verification at White Sands Space Harbor. IEEE Transactions on Geoscience \& Remote Sensing 45(1), 147-155.

Schutz, B.E., 2001. GLAS Altimeter Post-Launch Calibration/Validation Plan. Center for Space Research University of Texas at Austin, Austin, USA.

Takaku, J., Tadono, T., Tsutsui, K., 2016a. Generation of high resolution global DSM from ALOS PRISM. ISPRS International Archives of the Photogrammetry, Remote Sensing and Spatial Information Sciences.

Takaku, J., Tadono, T., Tsutsui, K., Ichikawa, M., 2016b. VALIDATION OF 'AW3D' GLOBAL DSM GENERATED FROM ALOS PRISM. ISPRS Annals of the Photogrammetry, Remote Sensing and Spatial Information Sciences III-4, 25-31.

Tang, X., Xie, J., Gao, X., Mo, F., Feng, W., Liu, R., 2019a. The In-Orbit Calibration Method Based on Terrain Matching With Pyramid-Search for the Spaceborne Laser Altimeter. IEEE Journal of Selected Topics in Applied Earth Observations and Remote Sensing 12(3), 1053-1062.

Tang, X., Xie, J., Liu, R., Huang, G., Zhao, C., Zhen, Y., Tang, H., Dou, X., 2019b. Overview of the GF-7 Laser Altimeter System Mission. Earth and Space Science 6(11), 1-11.

Xie, J., Tang, X., Mo, F., Tang, H., Wang, Z., Wang, X., Liu, Y., Tian, S., Liu, R., Xia, X., 2018. In-orbit geometric calibration and experimental verification of the ZY3-02 laser altimeter. The Photogrammetric Record 33(163), 341-362.

Yi Hong, L.S., Ma Yue, Huang Ke, Zhou Hui, Shi Guang-Yuan, 2017. On-orbit calibration of satellite laser altimeters based on footprint detection. Acta Physica Sinica 66(13), 134206. 